\title{
New Alternately Linearized Implicit Iteration for M-matrix Algebraic Riccati Equations
}

\author{
Jinrui Guan ${ }^{1, *}$ and Linzhang $\mathrm{Lu}^{2}$ \\ ${ }^{1}$ Department of Mathematics, Taiyuan Normal University, Shanxi 030619, \\ P.R. China. \\ 2 School of Mathematics and Computer Science, Guizhou Normal University, \\ Guizhou 550001, P.R. China.
}

Received October 16, 2015; Accepted May 17, 2016

\begin{abstract}
Research on the theories and the efficient numerical methods of M-matrix algebraic Riccati equation (MARE) has become a hot topic in recent years due to its broad applications. In this paper, based on the alternately linearized implicit iteration method (ALI) [Z.-Z. Bai et al., Numer. Linear Algebra Appl., 13(2006), 655-674.], we propose a new alternately linearized implicit iteration method (NALI) for computing the minimal nonnegative solution of M-matrix algebraic Riccati equation. Convergence of the NALI method is proved by choosing proper parameters for the MARE associated with nonsingular M-matrix or irreducible singular M-matrix. Theoretical analysis and numerical experiments show that the NALI method is more efficient than the ALI method in some cases.
\end{abstract}

AMS subject classifications: 15A24, 65F30

Key words: M-matrix algebraic Riccati equation, M-matrix, ALI iteration method.

\section{Introduction}

The nonsymmetric algebraic Riccati equation (NARE) is of the form

$$
X C X-X D-A X+B=0,
$$

where $A, B, C$ and $D$ are real matrices of sizes $m \times m, m \times n, n \times m$ and $n \times n$ respectively. For (1.1), let

$$
K=\left(\begin{array}{cc}
D & -C \\
-B & A
\end{array}\right)
$$

If $K$ is an M-matrix, then (1.1) is called an M-matrix algebraic Riccati equation (MARE). M-matrix algebraic Riccati equation arises from many branches of applied mathematics,

${ }^{*}$ Corresponding author. Email addresses: guanjinrui2012@163.com (J. Guan), 1lz@gznu.edu.cn (L. Lu) 
such as transport theory, Wiener-Hopf factorization of Markov chains, stochastic process, and so on $[2,3,5,7,14,18,20]$. Research on the theories and the efficient numerical methods of MARE has become a hot topic in recent years. The solution of practical interest is the minimal nonnegative solution. For theoretical background we refer to $[5,7,8,10-12,15]$.

The following are some notations and definitions we need in the sequel.

For any matrices $A=\left(a_{i j}\right), B=\left(b_{i j}\right) \in \mathbb{R}^{m \times n}$, we write $A \geq B(A>B)$, if $a_{i j} \geq b_{i j}\left(a_{i j}>b_{i j}\right)$ for all $i, j$. $A$ is called a Z-matrix if $a_{i j} \leq 0$ for all $i \neq j$. A Z-matrix $A$ is called an M-matrix if there exists a nonnegative matrix $B$ such that $A=s I-B$ and $s \geq \rho(B)$ where $\rho(B)$ is the spectral radius of $B$. In particular, $A$ is called a nonsingular M-matrix if $s>\rho(B)$ and singular M-matrix if $s=\rho(B)$.

We review some basic results of M-matrix. The following lemmas can be found in [4, Chapter 6].

Lemma 1.1. Let $A$ be a Z-matrix. Then the following statements are equivalent:

(1) $A$ is a nonsingular M-matrix;

(2) $A^{-1} \geq 0$;

(3) $A v>0$ for some vectors $v>0$;

(4) All eigenvalues of A have positive real part.

Lemma 1.2. Let $A$ and $B$ be Z-matrices. If $A$ is a nonsingular $M$-matrix and $A \leq B$, then $B$ is also a nonsingular M-matrix. In particular, for any nonnegative real number $\alpha, B=\alpha I+A$ is a nonsingular M-matrix.

Lemma 1.3. Let $A$ be an $M$-matrix, $B \geq A$ be a $Z$-matrix. If $A$ is nonsingular or irreducible singular and if $A \neq B$, then $B$ is also a nonsingular M-matrix.

Lemma 1.4. Let $A$ be a nonsingular M-matrix or an irreducible singular M-matrix. Let $A$ be partitioned as

$$
A=\left(\begin{array}{ll}
A_{11} & A_{12} \\
A_{21} & A_{22}
\end{array}\right),
$$

where $A_{11}$ and $A_{22}$ are square matrices. Then $A_{11}$ and $A_{22}$ are nonsingular M-matrices.

Lemma 1.5. If $A, B$ are nonsingular M-matrices and $A \leq B$, then $A^{-1} \geq B^{-1}$.

For the minimal nonnegative solution of the MARE, we have the following important result $[5,7,8,12]$.

Lemma 1.6. If $K$ is a nonsingular M-matrix or an irreducible singular M-matrix, then (1.1) has a unique minimal nonnegative solution $S$. If $K$ is nonsingular, then $A-S C$ and $D-C S$ are also nonsingular $M$-matrices. If $K$ is irreducible, then $S>0$ and $A-S C$ and $D-C S$ are also irreducible M-matrices.

There are many numerical methods up to now proposed for the minimal nonnegative solution of MARE, such as Schur method, matrix sign function, fixed-point iteration, Newton iteration, doubling algorithms, and so on. For details see $[1,5-7,9,13,16,17,19]$. 
For the MARE with a nonsingular M-matrix, the alternately linearized implicit iteration method (ALI) was proposed in [1] as follows.

\section{The ALI method}

- Set $X_{0}=0 \in \mathbb{R}^{m \times n}$.

- For $k=0,1, \cdots$, until $\left\{X_{k}\right\}$ converges, compute $X_{k+1}$ from $X_{k}$ by solving the following two systems of linear matrix equations:

$$
\left\{\begin{array}{l}
X_{k+1 / 2}\left(\alpha I+\left(D-C X_{k}\right)\right)=(\alpha I-A) X_{k}+B \\
\left(\alpha I+\left(A-X_{k+1 / 2} C\right)\right) X_{k+1}=X_{k+1 / 2}(\alpha I-D)+B
\end{array}\right.
$$

where $\alpha>0$ is a given parameter.

The following convergence theorem of the ALI method was proved in [1].

Theorem 1.1. For the MARE (1.1), if the matrix $K$ in (1.2) is a nonsingular M-matrix and the parameter $\alpha$ satisfies

$$
\alpha \geq \max \left\{\max _{1 \leq i \leq m}\left\{a_{i i}\right\}, \max _{1 \leq j \leq n}\left\{d_{j j}\right\}\right\},
$$

then $\left\{X_{k}\right\}$ generated by the ALI method is well defined, monotonically increasing and converges to $S$, the minimal nonnegative solution.

In the methods of Newton and fixed-point, it needs to solve a Sylvester matrix equation in each iteration, while in the ALI method it only needs to solve two systems of linear matrix equations in each iteration. Since it is much easier to solve linear matrix equation than Sylvester matrix equation, the ALI method is effective than the Newton method and fixed-point methods.

However, there is still room for improvement in the ALI method. In this paper, we propose a new alternately linearized implicit iteration method (NALI) for solving the minimal nonnegative solution of MARE. Compared with the ALI method, the new iteration method is more efficient because the coefficient matrices of the linear matrix equations in NALI are fixed in each iteration. Hence, less CPU times are required for solving MARE, which will be confirmed by the numerical results.

The rest of the paper is organized as follows. In section 2, we propose a new ALI method and prove its convergence. In Section 3, we use some numerical examples to show the effectiveness of the new method. Concluding remarks is given in Section 4.

\section{New ALI method}

In the following we propose a new ALI method (NALI). 


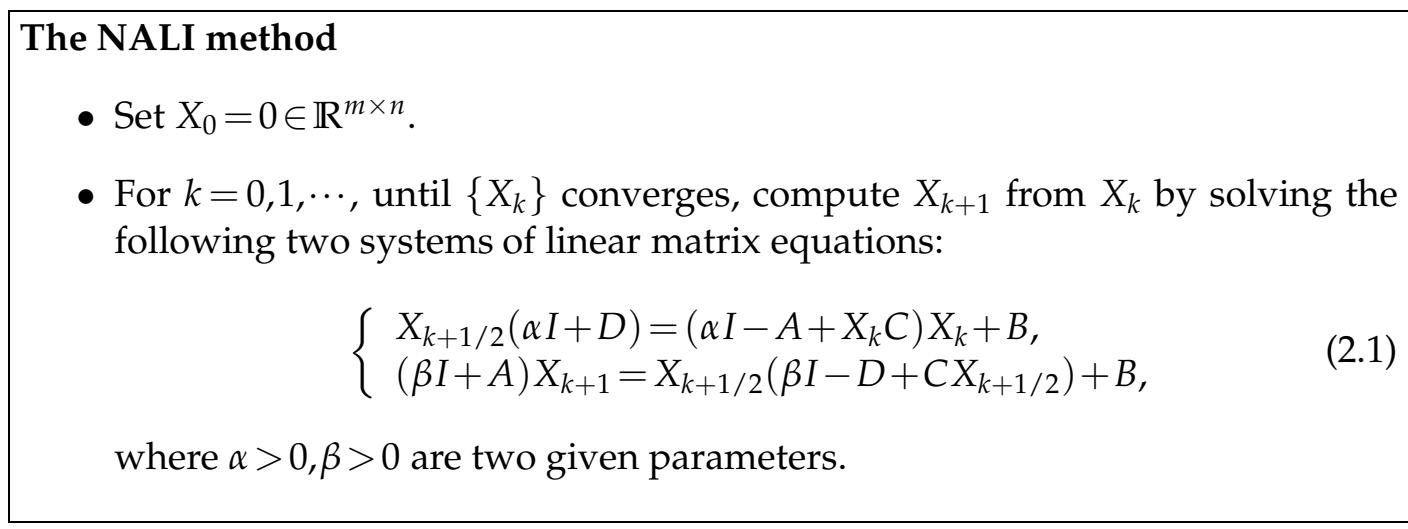

The NALI method has the same coefficient matrices in the linear matrix equations as the ALI method, but independent of the iteration step. Compared with the ALI method, the NALI method is more efficient since the coefficient matrices of the linear matrix equations are fixed in each iteration. Hence, less CPU times are required for solving MARE.

In the following, we give the convergence analysis of the NALI method. We need several lemmas.

Lemma 2.1. Let $\left\{X_{k}\right\}$ be the matrix sequence generated by the NALI method,

$$
\mathcal{R}(X)=X C X-X D-A X+B,
$$

and $S$ be the minimal nonnegative solution to (1.1). Then for any $k \geq 0$, the following equalities hold:

(a) $\left(X_{k+1 / 2}-S\right)(\alpha I+D)=\left(\alpha I-A+X_{k} C\right)\left(X_{k}-S\right)+\left(X_{k}-S\right) C S$;

(b) $\left(X_{k+1 / 2}-X_{k}\right)(\alpha I+D)=\mathcal{R}\left(X_{k}\right)$;

(c) $\mathcal{R}\left(X_{k+1 / 2}\right)=\left(\alpha I-A+X_{k+1 / 2} C\right)\left(X_{k+1 / 2}-X_{k}\right)+\left(X_{k+1 / 2}-X_{k}\right) C X_{k}$;

(d) $(\beta I+A)\left(X_{k+1}-S\right)=\left(X_{k+1 / 2}-S\right)\left(\beta I-D+C X_{k+1 / 2}\right)+S C\left(X_{k+1 / 2}-S\right)$;

(e) $(\beta I+A)\left(X_{k+1}-X_{k+1 / 2}\right)=\mathcal{R}\left(X_{k+1 / 2}\right)$;

(f) $\mathcal{R}\left(X_{k+1}\right)=\left(X_{k+1}-X_{k+1 / 2}\right)\left(\beta I-D+C X_{k+1}\right)+X_{k+1 / 2} C\left(X_{k+1}-X_{k+1 / 2}\right)$.

Proof. We first prove (a). From $S C S-S D-A S+B=0$, we have $-S D=A S-S C S-B$. Thus

$$
\begin{aligned}
& \left(X_{k+1 / 2}-S\right)(\alpha I+D)=X_{k+1 / 2}(\alpha I+D)-S(\alpha I+D) \\
= & \left(\alpha I-\left(A-X_{k} C\right)\right) X_{k}+B-\alpha S+(A S-S C S-B) \\
= & \left(\alpha I-A+X_{k} C\right)\left(X_{k}-S\right)+\left(X_{k}-S\right) C S .
\end{aligned}
$$


Next we prove (b) and (c). We have

$$
\begin{aligned}
& \left(X_{k+1 / 2}-X_{k}\right)(\alpha I+D)=X_{k+1 / 2}(\alpha I+D)-X_{k}(\alpha I+D) \\
= & \left(\alpha I-\left(A-X_{k} C\right)\right) X_{k}+B-X_{k}(\alpha I+D)=\mathcal{R}\left(X_{k}\right) .
\end{aligned}
$$

From (2.1), we have

$$
X_{k+1 / 2} D=\left(\alpha I-\left(A-X_{k} C\right)\right) X_{k}+B-\alpha X_{k+1 / 2} .
$$

Thus

$$
\begin{aligned}
& \mathcal{R}\left(X_{k+1 / 2}\right)=X_{k+1 / 2} C X_{k+1 / 2}-A X_{k+1 / 2}-X_{k+1 / 2} D+B \\
= & \left(X_{k+1 / 2} C X_{k+1 / 2}-A X_{k+1 / 2}+B\right)-\left(\left(\alpha I-A+X_{k} C\right) X_{k}+B-\alpha X_{k+1 / 2}\right) \\
= & \left(\alpha I-A+X_{k+1 / 2} C\right)\left(X_{k+1 / 2}-X_{k}\right)+\left(X_{k+1 / 2}-X_{k}\right) C X_{k} .
\end{aligned}
$$

Finally, we prove (d), (e) and (f). We have

$$
\begin{aligned}
& (\beta I+A)\left(X_{k+1}-S\right)=(\beta I+A) X_{k+1}-(\beta I+A) S \\
& \quad=X_{k+1 / 2}\left(\beta I-\left(D-C X_{k+1 / 2}\right)\right)+B-\beta S-(S D-S C S-B) \\
& \quad=\left(X_{k+1 / 2}-S\right)\left(\beta I-D+C X_{k+1 / 2}\right)+S C\left(X_{k+1 / 2}-S\right) \\
& (\beta I+A)\left(X_{k+1}-X_{k+1 / 2}\right)=(\beta I+A) X_{k+1}-(\beta I+A) X_{k+1 / 2} \\
& \quad=X_{k+1 / 2}\left(\beta I-\left(D-C X_{k+1 / 2}\right)\right)+B-(\beta I+A) X_{k+1 / 2}=\mathcal{R}\left(X_{k+1 / 2}\right), \\
& \mathcal{R}\left(X_{k+1}\right)=X_{k+1} C X_{k+1}-A X_{k+1}-X_{k+1} D+B \\
& \quad=\left(X_{k+1} C X_{k+1}-X_{k+1} D+B\right)-\left(X_{k+1 / 2}\left(\beta I-\left(D-C X_{k+1 / 2}\right)\right)+B+\beta X_{k+1}\right) \\
& \quad=\left(X_{k+1}-X_{k+1 / 2}\right)\left(\beta I-D+C X_{k+1}\right)+X_{k+1 / 2} C\left(X_{k+1}-X_{k+1 / 2}\right) .
\end{aligned}
$$

This completes the proof of the lemma.

Lemma 2.2. Let $\left\{X_{k}\right\}$ be the matrix sequence generated by the NALI method, $K$ in (1.2) be a nonsingular M-matrix or an irreducible singular M-matrix and $S$ be the minimal nonnegative solution to (1.1). If the parameters $\alpha, \beta$ of the NALI method satisfy

$$
\alpha \geq \max \left\{a_{i i}\right\}, \quad \beta \geq \max \left\{d_{j j}\right\},
$$

then for any $k \geq 0$,

$$
0 \leq X_{k+1 / 2} \leq S, \quad 0 \leq X_{k+1} \leq S .
$$

Proof. When $K$ in (1.2) is a nonsingular M-matrix or an irreducible singular M-matrix, $A$ and $D$ are nonsingular M-matrices by Lemma 1.4. Thus $\alpha I+D$ and $\beta I+A$ are both nonsingular M-matrices by Lemma 1.2. From Lemma 1.1 we have $(\alpha I+D)^{-1} \geq 0,(\beta I+$ $A)^{-1} \geq 0$.

We prove (2.2) by induction. When $k=0$, from (2.1) we have $X_{1 / 2}(\alpha I+D)=B$. Thus

$$
X_{1 / 2}=B(\alpha I+D)^{-1} \geq 0 .
$$


On the other hand, by Lemma 2.1(1), we have

$$
\left(X_{1 / 2}-S\right)(\alpha I+D)=(\alpha I-A)(-S)-S C S \leq 0,
$$

thus

$$
X_{1 / 2}-S=((\alpha I-A)(-S)-S C S)(\alpha I+D)^{-1} \leq 0 .
$$

Similarly, from (2.1) we have

$$
(\beta I+A) X_{1}=X_{1 / 2}\left(\beta I-D+C X_{1 / 2}\right)+B,
$$

thus

$$
X_{1}=(\beta I+A)^{-1}\left(X_{1 / 2}\left(\beta I-D+C X_{1 / 2}\right)+B\right) \geq 0 .
$$

From Lemma 2.1(4), we have

$$
(\beta I+A)\left(X_{1}-S\right)=\left(X_{1 / 2}-S\right)\left(\beta I-D+C X_{1 / 2}\right)+S C\left(X_{1 / 2}-S\right),
$$

thus

$$
X_{1}-S=(\beta I+A)^{-1}\left(\left(X_{1 / 2}-S\right)\left(\beta I-D+C X_{1 / 2}\right)+S C\left(X_{1 / 2}-S\right)\right) \leq 0 .
$$

Thus we have proved

$$
0 \leq X_{1 / 2} \leq S, \quad 0 \leq X_{1} \leq S .
$$

Suppose that the assertions (2.2) hold for $k=l-1$. From (2.1), we have

$$
X_{l+1 / 2}(\alpha I+D)=\left(\alpha I-\left(A-X_{l} C\right)\right) X_{l}+B,
$$

thus

$$
X_{l+1 / 2}=\left(\left(\alpha I-A+X_{l} C\right) X_{l}+B\right)(\alpha I+D)^{-1} \geq 0 .
$$

On the other hand, by Lemma 2.1(1), we have

$$
\left(X_{l+1 / 2}-S\right)(\alpha I+D)=\left(\alpha I-A+X_{l} C\right)\left(X_{l}-S\right)+\left(X_{l}-S\right) C S,
$$

thus

$$
X_{l+1 / 2}-S=\left(\left(\alpha I-A+X_{l} C\right)\left(X_{l}-S\right)+\left(X_{l}-S\right) C S\right)(\alpha I+D)^{-1} \leq 0 .
$$

Similarly, from (2.1) we have

$$
(\beta I+A) X_{l+1}=X_{l+1 / 2}\left(\beta I-\left(D-C X_{l+1 / 2}\right)\right)+B,
$$

thus

$$
X_{l+1}=(\beta I+A)^{-1}\left(X_{l+1 / 2}\left(\beta I-D+C X_{l+1 / 2}\right)+B\right) \geq 0 .
$$

On the other hand, by Lemma 2.1(4), we have

$$
(\beta I+A)\left(X_{l+1}-S\right)=\left(X_{l+1 / 2}-S\right)\left(\beta I-D+C X_{l+1 / 2}\right)+S C\left(X_{l+1 / 2}-S\right),
$$

thus

$$
X_{l+1}-S=(\beta I+A)^{-1}\left(\left(X_{l+1 / 2}-S\right)\left(\beta I-D+C X_{l+1 / 2}\right)+S C\left(X_{l+1 / 2}-S\right)\right) \leq 0 .
$$

Hence the assertions (2.2) hold for $k=l$.

Thus we have proved by induction that the assertions (2.2) hold for all $k \geq 0$. 
Lemma 2.3. Let the assumption be as in Lemma 2.2. Then for any $k \geq 0$, we have

$$
X_{k} \leq X_{k+1 / 2} \leq X_{k+1}, \quad \mathcal{R}\left(X_{k+1 / 2}\right) \geq 0, \quad \mathcal{R}\left(X_{k+1}\right) \geq 0 .
$$

Proof. We prove this by induction. When $k=0$, it is clear that $0=X_{0} \leq X_{1 / 2}$. From Lemma 2.1(3), we have

$$
\mathcal{R}\left(X_{1 / 2}\right)=\left(\alpha I-A+X_{1 / 2} C\right) X_{1 / 2} \geq 0 .
$$

By Lemma 2.1(5), we have $(\beta I+A)\left(X_{1}-X_{1 / 2}\right)=\mathcal{R}\left(X_{1 / 2}\right)$. Thus

$$
X_{1}-X_{1 / 2}=(\beta I+A)^{-1} \mathcal{R}\left(X_{1 / 2}\right) \geq 0 .
$$

By Lemma 2.1(6), we have

$$
\mathcal{R}\left(X_{1}\right)=\left(X_{1}-X_{1 / 2}\right)\left(\beta I-D+C X_{1}\right)+X_{1 / 2} C\left(X_{1}-X_{1 / 2}\right) \geq 0 .
$$

Suppose that the assertions hold for $k=l-1$. By Lemma 2.1(2), we have $\left(X_{l+1 / 2}-X_{l}\right)(\alpha I+$ $D)=\mathcal{R}\left(X_{l}\right)$. Thus

$$
X_{l+1 / 2}-X_{l}=\mathcal{R}\left(X_{l}\right)(\alpha I+D)^{-1} \geq 0 .
$$

From Lemma 2.1(3), we have

$$
\mathcal{R}\left(X_{l+1 / 2}\right)=\left(\alpha I-A+X_{l+1 / 2} C\right)\left(X_{l+1 / 2}-X_{l}\right)+\left(X_{l+1 / 2}-X_{l}\right) C X_{l} \geq 0 .
$$

From Lemma 2.1(5), we have $(\beta I+A)\left(X_{l+1}-X_{l+1 / 2}\right)=\mathcal{R}\left(X_{l+1 / 2}\right)$. Thus

$$
X_{l+1}-X_{l+1 / 2}=(\beta I+A)^{-1} \mathcal{R}\left(X_{l+1 / 2}\right) \geq 0 .
$$

By Lemma 2.1(6), we have

$$
\mathcal{R}\left(X_{l+1}\right)=\left(X_{l+1}-X_{l+1 / 2}\right)\left(\beta I-D+C X_{l+1}\right)+X_{l+1 / 2} C\left(X_{k l+1}-X_{l+1 / 2}\right) \geq 0 .
$$

Hence the assertions hold for $k=l$.

Thus we have proved by induction that the assertions hold for all $k \geq 0$.

Using the lemmas above, we can prove the following convergence theorem of the NALI method.

Theorem 2.1. For the MARE (1.1), if $K$ in (1.2) is a nonsingular M-matrix or an irreducible singular M-matrix and the parameters $\alpha, \beta$ satisfy

$$
\alpha \geq \max \left\{a_{i i}\right\}, \quad \beta \geq \max \left\{d_{j j}\right\},
$$

then $\left\{X_{k}\right\}$, generated by the NALI method, is well defined, monotonically increasing and converges to $S$, the minimal nonnegative solution. 
Proof. Combining Lemma 2.2 with Lemma 2.3, we have shown that $\left\{X_{k}\right\}$ is nonnegative, monotonically increasing and bounded from above. Thus there is a nonnegative matrix $S^{*}$ such that $\lim _{k \rightarrow \infty} X_{k}=S^{*}$. It also holds that $\lim _{k \rightarrow \infty} X_{k+1 / 2}=S^{*}$. From Lemma 2.2, we have $S^{*} \leq S$. On the other hand, take the limit in the NALI method, we know $S^{*}$ is a solution of the MARE (1.1), thus $S \leq S^{*}$. Hence $S=S^{*}$.

In actual computations, we choose the parameters in the NALI method to be

$$
\alpha=\max \left\{a_{i i}\right\}, \quad \beta=\max \left\{d_{j j}\right\} .
$$

To solve the linear matrix equations in the NALI method efficiently, we first compute the inverse of the coefficient matrices when the size of the problem is not too large.

\section{Numerical experiments}

In this section we use several examples to show the effectiveness of the NALI method. We compare the NALI method with the ALI method and present computational results in terms of the numbers of iterations (IT), CPU time (CPU) and the residue (RES). The residue is defined to be

$$
R E S:=\frac{\|X C X-X D-A X+B\|_{\infty}}{\|X C X\|_{\infty}+\|X D\|_{\infty}+\|A X\|_{\infty}+\|B\|_{\infty}}
$$

as in [1]. In our implementations all iterations are run in MATLAB2012 on a personal computer and are terminated when the current iterate satisfies $R E S<10^{-6}$ or the number of iterations is more than 9000 .

Experiment 1. Consider the MARE (1.1) with

$$
\begin{array}{lll}
A=\left(\begin{array}{cc}
4.27 & -2 \\
-1 & 6
\end{array}\right), & B=\left(\begin{array}{cc}
1 & 1 \\
2 & 1
\end{array}\right), \\
C=\left(\begin{array}{ll}
3 & 4 \\
2 & 1
\end{array}\right), & D=\left(\begin{array}{cc}
5 & -1 \\
-1 & 4
\end{array}\right) .
\end{array}
$$

This MARE is taken from [6], where the corresponding $K$ is a nonsingular M-matrix. The computational results are summarized in Table 1. From Table 1 we can see that, though

Table 1: Computational results of Experiment 1.

\begin{tabular}{||cccc||}
\hline Methods & IT & CPU & RES \\
\hline ALI & 125 & 0.008192 & $9.8169 \mathrm{e}-07$ \\
NALI & 183 & 0.003300 & $9.6837 \mathrm{e}-07$ \\
\hline
\end{tabular}

the NALI method needs a little more iterations, it needs fewer CPU time than the ALI method. Thus the NALI method is more efficient than the ALI method. 
Experiment 2. Consider the MARE (1.1) with

$$
\begin{array}{ll}
D=\left(\begin{array}{cc}
2 & 0 \\
0 & 100
\end{array}\right), & C=\left(\begin{array}{ccc}
2 & 0 & 0 \\
100 & 0 & 0
\end{array}\right), \\
B=\left(\begin{array}{cc}
0 & 0 \\
0 & 0 \\
1.5 & 1.5
\end{array}\right), & A=\left(\begin{array}{ccc}
3 & -3 & 0 \\
0 & 3 & -3 \\
0 & 0 & 3
\end{array}\right) .
\end{array}
$$

This MARE is taken from [3], where the corresponding $K$ is an irreducible singular $M-$ matrix. The computational results are summarized in Table 2. From Table 2 we can see

Table 2: Computational results of Experiment 2.

\begin{tabular}{||cccc||}
\hline Methods & IT & CPU & RES \\
\hline ALI & 322 & 0.025949 & $9.9686 \mathrm{e}-07$ \\
NALI & 26 & 0.001307 & $6.5227 \mathrm{e}-07$ \\
\hline
\end{tabular}

that the NALI method is more efficient than the ALI method.

Experiment 3. Consider the MARE (1.1) with

$$
\begin{array}{lll}
D=\left(\begin{array}{cc}
30 & -10 \\
-10 & 30
\end{array}\right), & C=\left(\begin{array}{cc}
10 & 10 \\
10 & 10
\end{array}\right), \\
B=\left(\begin{array}{cc}
10 & 10 \\
10 & 10
\end{array}\right), & A=\left(\begin{array}{cc}
30 & -10 \\
-10 & 30
\end{array}\right) .
\end{array}
$$

This M-MARE is taken from [3] and the corresponding $K$ is an irreducible singular $M$ matrix. The computational results are summarized in Table 3. From Table 3 we can see

Table 3: Computational results of Experiment 3.

\begin{tabular}{||cccc||}
\hline Methods & IT & CPU & RES \\
\hline ALI & 375 & 0.025187 & $9.9800 \mathrm{e}-07$ \\
NALI & 622 & 0.022169 & $9.9718 \mathrm{e}-07$ \\
\hline
\end{tabular}

that, though with more iterations, the NALI method needs fewer CPU time than the ALI method, so it is efficient.

Experiment 4. Consider the MARE (1.1) for which $A, B, C, D$ are generated as follows.

First set $R=\operatorname{rand}(100,100)$; then set $W=\operatorname{diag}(\operatorname{Re})-R$, with $e=(1,1, \cdots, 1)^{T}$; finally, define

$$
\begin{array}{ll}
D=W(1: 50,1: 50), & C=-W(1: 50,51: 100), \\
B=-W(51: 100,1: 50), & A=W(51: 100,51: 100) .
\end{array}
$$


Table 4: Computational results of Experiment 4.

\begin{tabular}{||cccc||}
\hline Methods & IT & CPU & RES \\
\hline ALI & 22 & 0.029371 & $9.2745 \mathrm{e}-07$ \\
NALI & 29 & 0.019395 & $6.9917 \mathrm{e}-07$ \\
\hline
\end{tabular}

In this case, the corresponding $K$ is an irreducible singular M-matrix. The computational results are summarized in Table 4 . From Table 4 we can see that, though with a little more iterations, the NALI method needs fewer CPU time than the ALI method, so it is more efficient.

\section{Concluding remarks}

We have proposed a new alternately linearized implicit iteration method (NALI) for computing the the minimal nonnegative solution of the M-matrix algebraic Riccati equation. Convergence of the NALI method is guaranteed for the MARE associated with a nonsingular M-matrix or an irreducible singular M-matrix. Numerical experiments have shown that the NALI method is more efficient than the ALI method in some cases.

\section{Acknowledgments}

The authors would like to thank the anonymous referees and Prof. Ren-Cang Li for their kindly suggestions and comments, which greatly improved the original manuscript of this paper. This work was supported in part by National Natural Science Foundation of China (11671105) and Natural Science Foundation of China (11401424).

\section{References}

[1] Z.-Z. Bai, X.-X. Guo, S.-F. Xu. Alternately linearized implicit iteration methods for the minimal nonnegative solutions of the nonsymmetric algebraic Riccati equations. Numer. Linear Algebra Appl., 2006, 13: 655-674.

[2] N. G. Bean, M. M. O'Reilly, P. G. Taylor. Algorithms for return probabilities for stochastic fluid flows, Stochastic Models. 2005, 21: 149-184.

[3] N. G. Bean, M. M. O'Reilly, P. G. Taylor. Algorithms for the Laplace-Stieltjes transforms of first return times for stochastic fluid flows. Methodol Comput Appl Probab, 2008, 10: 381408.

[4] A. Berman, R. J. Plemmons. Nonnegative matrices in the mathematical sciences. Academic Press, New York, 1994.

[5] D. A. Bini, B. Iannazzo, B. Meini. Numerical solution of algebraic Riccati equations. SIAM series on Fundamentals of Algorithms, Philadelphia, 2012.

[6] C.-H. Guo, A. J. Laub. On the iterative solution of a class of nonsymmtric algebraic Riccati equation. SIAM J. Matrix Anal. Appl., 2000, 22(2): 376-391. 
[7] C.-H. Guo. Nonsymmetric algebraic Riccati equations and Wiener-Hopf factorization for Mmatrices. SIAM J. Matrix Anal. Appl., 2001, 23: 225-242.

[8] C.-H. Guo. A note on the minimal nonnegative solution of a nonsymmtric algebraic Riccati equation. Linear Algebra Appl., 2002, 357: 299-302.

[9] C.-H. Guo, N. J. Higham. Iterative solution of a nonsymmtric algebraic Riccati equation. SIAM J. Matrix Anal. Appl.,2007, 29: 396-412.

[10] C.-H. Guo. A new class of nonsymmtric algebraic Riccati equations. Linear Algebra Appl., 2007, 426: 636-649.

[11] C.-H. Guo, B. Iannazzo, B. Meini. On the doubling algorithm for a (shift) algebraic Riccati equation. SIAM J. Matrix Anal. Appl., 2007, 29: 1083-1100.

[12] C.-H. Guo. On algebraic Riccati equations associated with M-matrices. Linear Algebra Appl., 2013, 439: 2800-2814.

[13] X.-X. Guo. Theories and algorithms for several nonlinear matrix equations, PhD Thesis. Chinese Academy of Sciences, China, 2005.

[14] J. Juang, W.-W. Lin. Nonsymmetric algebraic Riccati equations and Hamilton-like matrices. SIAM J. Matrix Anal. Appl., 1999, 20: 228-243.

[15] P. Lancaster, L. Rodman. Algebraic Riccati equations. Oxford University Press, Oxford, 1995.

[16] L.-Z. Lu. Solution form and simple iteration of a nonsymmetric algebraic Riccati equation arising in transport theory. SIAM J. Matrix Anal. Appl., 2005, 26: 679-685.

[17] V. Mehrmann. The Autonomous linear quadratic control problem. Lecture Notes in Control and Information Sciences, 163, Springer-Verlag, Berlin, 1991.

[18] L. C. G. Rogers. Fluid models in queueing theory and Wiener-Hopf factorization of Markov chains. Ann. Appl. Probab., 1994, 4: 390-413.

[19] W.-G. Wang, W.-C. Wang, R.-C. Li. Alternating-directional doubling algorithm for M-matrix algebraic Riccati equations. SIAM J. Matrix Anal. Appl., 2012, 33: 170-194.

[20] K.M. Zhou, P.P. Khargonekar. An algebraic Riccati equation approach to $H^{\infty}$ optimization. Systems Control Lett., 1988, 11: 85-91. 